\title{
LES CHIRONOMIDES (DIPTERA) D'UN TORRENT PYRENEEN DE HAUTE MONTAGNE : L'ESTARAGNE
}

\author{
par H. LAVILLE1 et P. LAVANDIER 1 .
}

61 espèces de Chironomides ont été capturées (à l'état imaginal et nymphal) clans le torrent froid d'Estaragne; leurs périodes de vol sont précisées. Sur les 33 espèces rhéobiontes du peuplement, 9 sont abondantes. Diamesa hamaticornis et Eukiefferiella calvescens prédominent sur l'ensemble du réseau hyidrographique.

Deux zones se distinguent de part et d'autre d'un ressaut glaciaire. La partie amont du torrent $(>2: 130 \mathrm{~m})$ présente les caractères d'un torrent glaciaire et une biocénose riche en Diamesini (6 espèces) caractérisée par Eukiefferiella fittkaui, Paratrichocladius skirwithensis, Diamesa zernyi. La partie aval (2050$1850 \mathrm{~m}$ ) présente les caractères d'un torrent de haute montagne riche en Eukiefferiella (4 espèces) et caractérisée par Eukiefferiella minor et Pseudodiamesa branickii.

\section{The chironomids of Estaragne, a Pyrenean torrential stream of the high mountains.}

61 chironomid species have been captured as adults or pupae from the cold terrential of Estaragne. Their flight periods are given. Nine of the 33 rheobiontic species are abundant, and Diamesa hamaticornis and Eukiefferiella calvescens predominate in the whole river system.

Two zones can be recognised on either side of a glacial rise. The upper part $(>2130 \mathrm{~m})$ shows the characteristics of a glacial torrent with a biocenosis rich in Diamesini (6 species), and characterised by Eukiefferiella fittkaui, Paratrichocladius skirwithensis, Diamesa zernyi. The lower part $(2050-1850 \mathrm{~m})$ shows the characteristics of a high mountain torrential stream, rich in Eukiefferiella (4 species) and characterised by Eukiefferiella minor and Pseudodiamesa branickii.

L'analyse du peuplement chironomidien de l'Estaragne a été conduite dans le cadre d'une étude écologique concernant l'ensemble des populations benthiques de ce torrent froid de haute montagne.

Les échantillonnages, réalisés durant trois années, ont permis la récolte d'un nombre conséquent de nymphes $(\mathrm{N})$, d'exuvies nymphales (E) et d'irnagos (I) sur tout le réseau hydrographique. Ce matériel s'avère le plus convenable pour une bonne connaissance spécifique des Chironomides grâce notamment aux importantes révisions systé-

1. Laborato:re d'Hy:drobiologie (ERA 702), Université Paul-Sabat:er, 118, route de Narbonne, 31077 Toulouse Cedex, France. 
matiques récentes concernant d'une part, la tribu des Diamesini (Serra-Tosio 1972, 1973) et d'autre part, le genre Eukiefferiella (Lehmann 1972) dont les larves colonisent abondamment les cours d'eau de montagne.

Les Chironomides des eaux courantes des Pyrénées sont très peu connus et la liste des espèces rhéobiontes, établie en majeure partie d'après du matériel larvaire récolté par Bertrand (1950 à 1956) est courte et déjà ancienne. Celle concernant les Diamesini a été récemment augmentée (Serra-Tosio, loc. cit.). L'inventaire des Chironomides Pyrénéens a été fait par l'un de nous (Laville, sous presse): il regroupe, outre les récoltes de Bertrand, des récoltes plus récentes effectuées d'est en ouest de la chaîne y compris celles du torrent d'Estaragne déterminées en juillet 1976.

En dehors de son apport faunistique, cette étude tente de préciser l'écologie des espèces dominantes et le déterminisme des biocénoses.

\section{1. - LE RESEAU HYDROGRAPHIQUE}

Le réseau hydrographique de l'Estaragne a été décrit en détail dans des travaux antérieurs (Lavandier 1974 ; Lavandier et Mur 1974). La figure I et le tableau I récapitulent la situation géographique et les principales caractéristiques physiographiques des localités prospectées pour cette étude.

Tableau I. - Caractéristiques principales des stations d'étude.

\begin{tabular}{|c|c|c|c|c|c|c|c|c|c|}
\hline & A & B & $\mathbf{R}$ & c & $\mathrm{D}$ & $a_{4}$ & $a_{i}$ & $\mathrm{a}_{6}$ & $a_{i}$ \\
\hline Altitude (en m.) & 2370 & 2190 & 2150 & 1920 & 1850 & 2150 & 1920 & 2050 & 1900 \\
\hline Température max. & 45 & 7 & 8,5 & 11 & 13 & 15 & 17 & 5 & 8 \\
\hline $\begin{array}{l}\text { Durée d'enneigement } \\
\text { (en mois) }\end{array}$ & $6-8$ & $5-7$ & $5-7$ & $4-6$ & $4-5$ & $5-6$ & $4-6$ & 46 & $4-6$ \\
\hline $\begin{array}{l}\text { Largeur moyenne } \\
\quad(\text { en } \mathrm{m})\end{array}$ & 1.2 & $2-3$ & $3-5$ & $3-5$ & 4.6 & $0,2-1$ & $0,5-1,5$ & 2 & $2-4$ \\
\hline Galets & +++ & +++ & +++ & +++ & $++t$ & + & + & + & \\
\hline Graviers & + & & + & + & ++ & + & ++ & + & \\
\hline Vase - Débris végétaux & & & & & & ++ & ++ & + & \\
\hline Mousses & & & & & & +++ & & +++ & ++ \\
\hline
\end{tabular}

Remarques :

- Sur l'ensemble du réseau, la durée d'enneigement varie avec l'altitude; par contre, la température dépend en grande partie du 


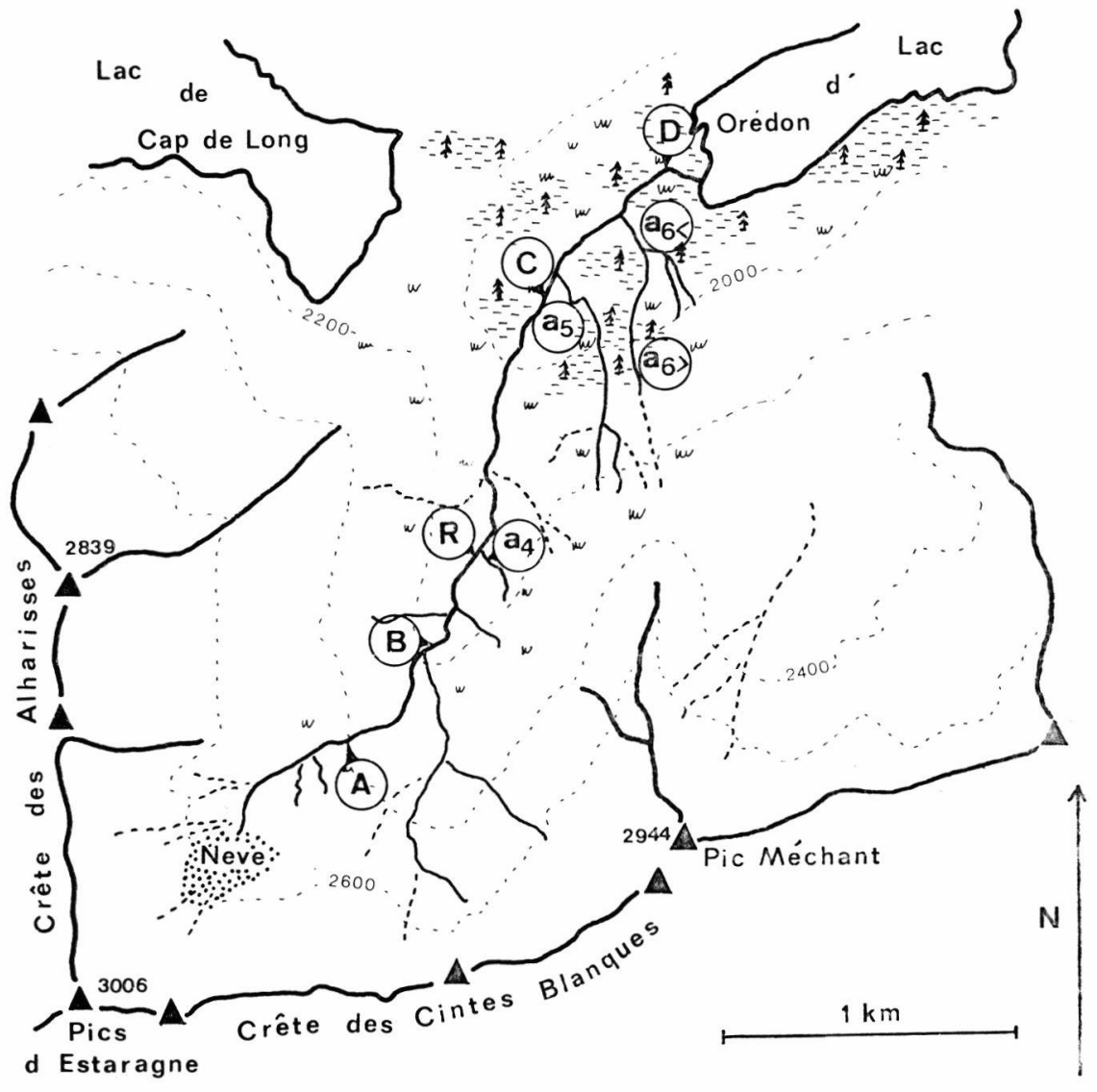

$w_{w}{ }^{w}$ Pelouse alpine Rhodopineraie

Fig. 1. - Carte de la zone étudiée.

mode d'alimentation et de la morphométrie des cours d'eau : ainsi le classement des stations en fonction de leurs températures maximales diffère-t-il de leur succession altitudinale.

- Tout au long de la période déneigée, le substrat du torrent principal se couvre périodiquement d'Hydrurus foetidus. Dans les affluents a4 et a6, ces algues n'apparaissent qu'au début de la fonte des neiges; elles se développent peu et disparaissent rapidement. Elles n'ont pas été observées dans le ruisseau a5.

- Plusieurs ruisselets tourbeux à Hypnacées rejoignent le torrent en amont de la station A. Près des stations $B$ et $R$ coulent des ruisselets riches en mousses : la proximité de ces milieux est à l'origine des captures parfois importantes d'espèces muscicoles. Les stations $\mathrm{a} 5$, a6 et surtout $\mathrm{D}$ sont situées dans une rhodopineraie claire. 


\section{2. - METHODES DE CAPTURE - PRESENTATION DES RESULTATS}

Les Chironomides n'ont pas fait l'objet de relevés particuliers. Le matériel examiné provient de récoltes intéressant tous les insectes aquatiques de l'Estaragne obtenus à partir de chasses en vol, de piégeages et de filets à dérive.

- Chasses en vol : en 1971 et 1972 une demi-heure de chasse hebdomadaire était effectuée à chaque station à l'aide d'un filet entomologique et d'un filet fauchoir ;

- Piègeages au cours de l'été 1972, 6 pièges à émergence ont été relevés régulièrement à la station $\mathrm{R}$.

- Filets à dérive: des filets ont été placés dans le courant aux différentes stations pendant la durée des chasses en vol. Plusieurs prélèvements proviennent de dérives effectuées sur 24 heures aux diverses stations d'étude.

Nous avons considéré séparément les espèces aquatiques principales et les espèces terrestres (sensu Strenzke 1950) ou rares en précisant dans le texte la totalité de l'effectif recueilli : $\delta, 8, \mathrm{~N} \hat{\delta}, \mathrm{E} \delta$ et $\mathrm{N} q, \mathrm{E} q$. Par contre, dans le tableau III qui indique la répartition des espèces dominantes, seuls les ô sont considérés, leur effectif étant comptabilisé sous forme de classe d'abondance. Cette méthode souple nous paraît la mieux adaptée, compte tenu des modalités et des difficultés de prélèvements. L'abondance des espèces peut être assimilée au nombre d'individus capturés à raison d'une heure de chasse hebdomadaire durant la période déneigée.

Dans le tableau II, les stations sont classées en fonction de leur température; mieux que l'étagement altitudinal, cette succession rend compte des similitudes et des dissemblances faunistiques existant entre les stations.

\section{3. - LE PEUPLEMENT}

\subsection{ESPECES PRINCIPALES}

Dans le torrent d'Estaragne et ses affluents, les Chironomides sont, par leur diversité, le principal composant de la faune benthique. Avec 58 espèces ils représentent plus du tiers du peuplement.

Thienemann $(1941,1954)$ caractérise les milieux d'eau courante arctique, semi-arctique et alpin par la prédominance des Orthocladiini et Diamesini : ceci est encore valable pour le torrent pyrénéen étudié où 6 Chironominae et 3 Tanypodinae seulement sont recensés. Le seul Chironomini présent, Polypedilum albicorne est une espèce sténotherme froide du crénon et de l'épirhithron (Lehmann 1971). 
Le tableau ci-dessous met en évidence la similitude de la composition spécifique des diverses sous-familles de Chironomides dans les torrents alpins de haute montagne (Thienemann 1954) et dans le torrent pyrénéen d'Estaragne.

$\begin{array}{lccccccccc} & \begin{array}{c}\text { Tany- } \\ \text { podinae } \\ \text { Nbre \% }\end{array} & \begin{array}{c}\text { Ortho- } \\ \text { cladinae } \\ \text { Nbre \% }\end{array} & \begin{array}{c}\text { Tanytarsini } \\ \text { Nbre } \%\end{array} & \begin{array}{c}\text { Chironomini } \\ \text { Nbre } \%\end{array} & \begin{array}{r}\text { Total } \\ \text { Nbre }\end{array} \\ \begin{array}{l}\text { Alpes } \\ \text { (Thienemann 1954) }\end{array} & 5 & 8 & 51 & 81 & 6 & 9,5 & 1 & 1,5 & 64 \\ \begin{array}{l}\text { Pyrénées } \\ \text { (Estaragne) }\end{array} & 3 & 5 & 49 & 84 & 5 & 8,5 & 1 & 1,5 & 58\end{array}$

La quasi-totalité des espèces sont des formes essentiellement rhéophiles. Seule, Parakiefferiella coronata a été précédemment trouvée en faciès lénitique dans la zone littorale de petits lacs du massif de Néouvielle avec parfois des densités de l'ordre de 1000 individus/m² (Laville 1972). Parmi les Tanytarsini, seule Micropsectra bidentata est fréquente et abondante tout le long du torrent.

Les Tanypodinae, Zavrelimyia barbatipes et Nilotanypus dubius ont été récoltés dans les affluents les plus chauds et les moins rapides ménageant des anses plus riches en sédiments propices à leur mode de vie. Il est intéressant de souligner la récolte d'une nymphe $q$ de Pentancura sp. Katterjokk dans la partie la plus froide issue du névé. Cette espèce a été récoltée par L. Brundin dans le Katterjokk à $1000 \mathrm{~m}$ d'altitude en Laponie suédoise (Fittkau 1962); la station de récolte pyrénéenne paraît confirmer sa sténothermie et témoigne d'une probable répartition boréoalpine.

Chez les Orthocladiinae, les Diamesini avec 10 espèces et les Eukiefferiella avec 7 espèces sont les plus diversifiées et les plus abondantes (fig. 2).

Parmi les 19 Metriocnemini, 6 représentent réellement le peuplement du torrent. Les autres, récoltés de façon plus épisodique, sont des espèces aux stades préimaginaux terrestres (sensu Strenzke 1950) ou colonisant des faciès hygropétriques.

Sur les 58 espèces recensées, 33 sont importantes dans le peuplement soit par leur abondance, soit par leur intérêt biogéographique : pour les Orthocladiinae, nous récapitulons les données écologiques et biogéographiques de la littérature par référence aux récents travaux de Lehmann $(1971,1972)$ et de Ringe (1974) dans des cours d'eau des Mittelgebirge d'Allemagne et à celui de Serra-Tosio (1973) pour la Tribu des Diamesini. Pour chaque espèce, nous donnons les récoltes globales ainsi que les périodes de vol entre $1850 \mathrm{~m}$ et $2340 \mathrm{~m}$.

La liste des espèces secondaires du peuplement est présentée à la suite avec les stations et les dates de récolte des imagos $\delta$. 


\section{ORTHOCLADIINAE}

\section{Diamesini}

\section{Pseudodiamesa branickii (Now.)}

Espèce à répartition boréoalpine holarctique.

Signalée dans les Alpes françaises entre $240 \mathrm{~m}$ et $2300 \mathrm{~m}$ où elle vit préférentiellement dans le Crénon et les mousses immergées mais aussi sur les pierres à couverture biologique mince à moyenne (SerraTosio 1973).

Récoltée dans le massif du Jura (Verneaux et Vergon 1976). Espèce à la fois rhéobionte et d'eau stagnante (Saether 1968).

Également trouvée dans les Pyrénées orientales dans la rivière la Lladure vers $1450 \mathrm{~m}$ (Laville, sous presse).

112 o ; 10 \%. Les imagos ont été récoltées fin juillet et mi-octobre à la station $\mathrm{D}(1850 \mathrm{~m})$ dont c'est une espèce dominante.

\section{Diamesa incallida (Walk.)}

Répartition paléarctique. France: Vosges, Alpes, Massif central et Pyrénées (Serra-Tosio 1973).

Espèce caractéristique des Sources (Eucrénon et Hypocrénon) dont la température peut s'élever jusqu'à près de $10^{\circ} \mathrm{C}$; elle n'est jamais dominante dans les sources au même titre que Pseudokiefferiella parva et Pseudodiamesa branickii (Serra-Tosio 1973).

Une seule imago ô récoltée sur la neige le 22-11-76 (Station R).

\section{Diamesa bertrami Edw.}

Espèce à très vaste répartition paléarctique : une des espèces de Diamesini les plus fréquentes des Alpes (entre 330 et $2360 \mathrm{~m}$ ) et de la bordure orientale du Massif Central à très basse altitude $(70 \mathrm{~m})$; en Espagne dans les monts cantabriques et la Sierra Nevada (SerraTosio 1973) ; Hautes-Pyrénées (Laville 1966). Espèce à large valence écologique (Euryöke) colonisant des eaux de température comprise entre $2^{\circ}$ et $14,5^{\circ} \mathrm{C}$.

Période de vol : fin août - mi-septembre (Stations $\mathrm{R}$ et a6 $<$ ).

9 s. 23 \% $1 \mathrm{~N}$ \%.

\section{Diamesa latitarsis (G.)}

Espèce à répartition boréoalpine, bien représentée dans la plupart des massifs montagneux d'Europe centrale (Serra-Tosio 1973).

Les stations connues des Alpes sont échelonnées entre $380 \mathrm{~m}$ et $2320 \mathrm{~m}$ : le maximum de fréquence se situe de 800 à $1500 \mathrm{~m}$. La larve colonise surtout les sources et l'Epirhithron où elle prédomine, plus exceptionnellement les torrents glaciaires (Serra-Tosio, loc. cit.). 
Signalée dans le Massif de Néouvielle à $2050 \mathrm{~m}$ et $2100 \mathrm{~m}$, entre $1100 \mathrm{~m}$ et $1300 \mathrm{~m}$ ainsi que dans les Pyrénées atlantiques au gave d'Ossau à $650 \mathrm{~m}$ (Serra-Tosio, loc. cit.).

Peu fréquente dans l'Estaragne: quelques imagos $\delta$ aux stations $\mathrm{B}, \mathrm{R}, \mathrm{D}$ en août et septembre. 27 fิ, $3 \% ; 11 \mathrm{~N}$ oे, $10 \mathrm{~N}$.

\section{Diamesa wuelkeri Serra-Tosio}

Répartition: Alpes et Pyrénées entre $1970 \mathrm{~m}$ et $2140 \mathrm{~m}$ (SerraTosio 1973). Dans les Alpes, elle est signalée dans des eaux de l'Hypocrénon dont les températures sont comprises entre $1,6^{\circ}$ et $11,6^{\circ} \mathrm{C}$.

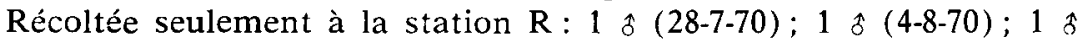
(13-8-71).

\section{Diamesa laticauda Serra-Tosio}

Répartition : Alpes françaises et Hauts-Tatras de Pologne ; elle est signalée dans les Alpes entre $1890 \mathrm{~m}$ et $2023 \mathrm{~m}$ dans des torrents glaciaires ou des ruisseaux de haute montagne (Serra-Tosio 1973). Alpes du Tyrol (Kownacka et Kownacki 1975). Diamesa nouvelle pour les Pyrénées.

Stations B et R: 4 of (7-14.8.70).

\section{Diamesa zernyi Edw.}

Espèce paléarctique à répartition médioeuropéenne orophile la plus fréquente dans les Alpes entre 300 et $2360 \mathrm{~m}$ (Serra-Tosio 1973).

Diamesa euryöke du Crénon à l'Hyporhithron. Connue des Pyrénées atlantiques au gave d'Aspe à $630 \mathrm{~m}$ (Serra-Tosio, loc. cit.). Espèce peu fréquente dans l'Estaragne mais présente de bas en haut du torrent. Déjà signalée au niveau de la station $\mathrm{D}$ : lac d'Orédon $(1850 \mathrm{~m}$ ) (Laville 1966: sub D. bohemani). 43 \&, 4 \%; $46 \mathrm{~N}$ \%.

\section{Diamesa cinerella (Mg.)}

Espèce orophile à répartition médioeuropéenne (alpine au sens large). Dans les Alpes et le Massif Central, la larve vit entre 100 et $2190 \mathrm{~m}$ du Crénon à l'Hyporhithron (Serra-Tosio 1973). Connue du Jura (Verneaux et Vergon 1976) et de la Neste d'Aure à $1570 \mathrm{~m}$ (Laville 1965).

Peu fréquente dans le torrent d'Estaragne: récoltée aux stations $\mathrm{A}, \mathrm{R}$ et $\mathrm{D}$. Egalement dans la rivière Lladure (Pyrénées orientales à $1480 \mathrm{~m}$ : Laville, sous presse).

Espèce euryöke (Kwnacka et Kownacki 1975) de même que $D$. Zernyi. 73 \&, 3 \% ; $17 \mathrm{E}$ ou $\mathrm{N}$ \&, $7 \mathrm{E}$ ou $\mathrm{N}$ \%.

\section{Diamesa hamaticornis $\mathrm{K}$.}

Espèce à répartition médioeuropéenne orophile. Elle vit dans l'épi- 
rhithron des cours d'eau du Sud-Est et du Massif Central de la France mais n'a jamais été trouvée dans les Alpes françaises (SerraTosio 1973). Très abondante dans la rivière Breitenbach d'avril à juillet dans la zone moyenne à truite (Ringe 1974).

Déjà signalée dans le Massif de Néouvielle du ruisseau de Lavedan $(1100-1300 \mathrm{~m})$ et dans les Pyrénées atlantiques au Gave de Pau (Serra-Tosio, loc. cit.). Récemment récoltée dans la Lladure (Pyrénées orientales) vers $1500 \mathrm{~m}$ (Laville, sous presse) et à $5 \mathrm{~km}$ de la source de la rivière Lot ( $1085 \mathrm{~m}$ : Tourenq, comm. verb.).

C'est le Diamesini dominant de l'Estaragne; sa période de vol dure 4 mois (mi-juillet - début novembre): on observe un décalage de près de 1 mois entre les premières imagos des stations basses $(C, D)$ et celles des stations hautes (A, B). Le maximum des émergences se

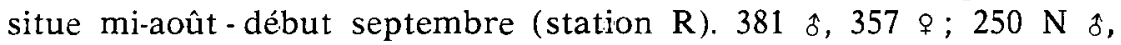
$475 \mathrm{~N}$ \%.

\section{Pseudokiefferiella parva (Edw.)}

Espèce holarctique, connue de la plupart des massifs montagneux d'Europe, de Grande-Bretagne, de Fennoscandinavie et du Canada (Serra-Tosio 1973). Dans les Alpes, la larve vit dans des eaux de 4,1 ${ }^{\circ}$ à $12,6^{\circ} \mathrm{C}$ du Crénon entre $720 \mathrm{~m}$ et $2350 \mathrm{~m}$ le plus souvent dans les mousses immergées (Serra-Tosio, loc. cit.).

4 o répartis dans les stations froides $\mathrm{A}, \mathrm{C}$ et a6 $>$.

\section{Orthocladiini}

\section{Cricotopus annulator $\mathrm{G}$.}

syn. : C. alpestris G. (Hirvenoja 1973).

Répartition : Toute l'Europe, ? Canada (Hirvenoja 1973); en France : Jura (Verneaux et Vergon 1976).

Vit essentiellement en eau courante et parfois dans les lacs (Hirvenoja, loc. cit.). Dans la Fulda depuis la source jusqu'à l'Hyporhithron, elle caractérise cependant le Rhithron (Lehmann 1971).

Dans les Pyrénées : d'Est en Ouest de la chaîne (Nivelle, Lissuraga dans les Pyrénées-Atlantiques; Lladure dans les Pyrénées-Orientales; cressonnière d'Agos dans les Pyrénées centrales: Laville, sous presse).

Dans le vallon d'Estaragne elle ne semble pas dépasser l'altitude de la station $D$ sinon dans les stations plus chaudes (a4 et a5).

27 s, 2 \%; $6 \mathrm{EN}$ \%.

\section{Eukiefferielia calvescens Edw.}

Répartition : Europe du Nord, du Centre et de l'Est (Lehmann 1972).

Espèce eurytope : la larve colonise la croûte algale des pierres en fort courant de la zone, à truite de la Fulda (Lehmann, loc. cit.). 
Très abondante dans la rivière Breitenbach (Ringe 1974) et dans un cours d'eau à Salmonidés du Jura (Verneaux et Vergon 1976).

Espèce la plus largement répandue sur tout le cours de l'Estaragne : elle prédomine à la station $B$. Longue période de vol : fin juin à fin octobre. Récemment trouvée dans les Pyrénées orientales : nivière la Lladure (1 440-1 $490 \mathrm{~m}$ ) (Tourenq, comm. verb.).

97 o, $59 q ; 140 \mathrm{~N}$ คे, $98 \mathrm{~N}$ \%.

\section{Eukiefferiella coerulescens $\mathrm{K}$.}

Répartition: Europe, Afrique du Nord (Lehmann 1971, 1972).

Dans la rivière Fulda, la larve vit sur les pierres et dans les mousses à fort courant du Crénon au Métarhithron. 7 fois moins fréquente que $E$. calvescens dans la rivière Breitenbach (Ringe 1974).

Récoltée seulement aux stations $\mathrm{C}$ et $\mathrm{R}$ et dans l'affluent a6 le plus riche en mousses. Période de vol : fin juillet-fin août (Station $\mathrm{C}$ ).

8 \&, 6 \%; $7 \mathrm{~N}$ \&.

\section{Eukiefferiella devonica Edw.}

Répartition : Connue sûrement de Laponie, de cours d'eau des Mittelgebirge d'Allemagne (Sauerland, Hessen) et d'Autriche (région de Lunz) (Lehmann 1972). Dans la Fulda, la larve vit dans les mousses à fort courant du Crénon à l'Hyporhithron (Lehmann 1971).

Peu fréquente dans l'Estaragne: quelques exemplaires dans les stations les plus chaudes $(C, D, a 5)$; imagos de mi-juillet à fin août.

$6 \mathrm{E} \hat{\delta}, 1 \mathrm{~N}$ o $; 6 \mathrm{E}$ \%, $9 \mathrm{~N}$ \%.

\section{Eukiefferiella fittkaui Lehmann}

Récemment différenciée de E. minor, E. fittkaui est connue d'Allemagne d'une source limnique de la Fulda et de cours d'eau des Mittelgebirge ; également signalée d'Autriche (région de Lunz) (Lehmann 1972).

C'est l'espèce dominante de la station A, la plus proche du névé; jamais récoltée en aval de la station $\mathbf{R}$, elle est à considérer plus sténotherme que E. minor présente surtout dans la partie basse du torrent.

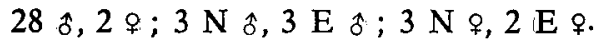

\section{Eukiefferiella fuldensis Lehmann}

L'exuvie nymphale diffère de celle de E. claripennis par la forme plus étroite et la taille moindre de la corne thoracique (Lehmann 1972). Cette différence n'est pas nette chez les spécimens de l'Estaragne: si la forme de la corne ressemble à celle de E. fuldensis, sa longueur varie avec le sexe; celle du of approche celle de $E$. claripennis $(0,2 \mathrm{~mm})$, celle de la $\uparrow(0,14 \mathrm{~mm})$ est plus proche de celle de 
E. fuldensis. Mais l'AR ô (ex pupa) $=0,45$ diffère nettement de celui de $E$. claripennis $(0,75-0,98)$ indiqué par Lehmann.

Seulement connue des Alpes (Partenkirchen) et de l'Epirhithron de la Fulda (Lehmann, loc. cit.).

Récoltée uniquement dans les stations les plus chaudes (C, D, a5); période de vol des imagos : fin juillet-fin août.

3 ô; $11 \mathrm{E} \hat{o} ; 16 \mathrm{E}$ \%.

\section{Eukiefferiella minor Edw.}

Répartition : Toute l'Europe (Lehmann 1972), Massif du Jura (Verneaux et Vergon 1976).

Dans la Fulda, depuis la source jusqu'à la zone moyenne à truite (Lehmann 1971). Eukiefferiella dominante de la rivière Breitenbach où elle vit davantage dans les mousses que sur les pierres (Ringe 1974).

Paraît vicariante avec E. fittkaui puisqu'elle n'apparaît que dans les stations basses; elles coexistent toutefois dans les stations intermédiaires $(B, R)$ mais y sont peu abondantes.

Période de vol : fin juillet et début actobre (Station $C$ ).

8 s., 12 \%; $3 \mathrm{~N}$ oै, $3 \mathrm{E}$ o ; $5 \mathrm{E}$ \%.

\section{Eukiefferiella tirolensis $G$.}

Répartition : R.F.A., Suisse, Autriche, France et Espagne : en France connue des Alpes, en Espagne de la Sierra Nevada (Lehmann 1972).

Uniquement récoltée à la station a6 $>$, la plus froide et la plus riche en mousses, $E$. tirolensis semble une espèce sténotherme froide.

Période de vol : fin juillet à fin août.

84 ô; $14 \mathrm{E}$ ô, $1 \mathrm{~N}$ \%, $2 \mathrm{E}$ \%.

\section{Euorthocladius rivicola $\mathrm{K}$.}

Répartition : Europe sauf le Sud (Lehmann 1971); Canada (Saether 1969).

Espèce fortement rhéobionte mais eurytherme connue depuis la source jusqu'au cours inférieur des cours d'eau (Dittmar 1955). Dans la Fulda sa plus grande abondance se situe dans le Rhithron (Lehmann, loc. cit.).

E. rivicola a été récoltée tout le long du torrent d'Estaragne dont elle est une des espèces dominantes. Les premières imagos apparaissent début juillet à la station $\mathrm{D}$ et seulement à la mi-août aux stations $\mathrm{B}$ et $\mathrm{A}$. La période d'émergence dure un mois dans les stations basses mais se prolonge jusqu'à fin octobre dans les stations hautes.

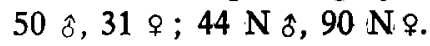




\title{
Paratrichocladius skirwithensis Edw.
}

\author{
syn.: Syncricotopus nivalis G. (Hirvenoja 1973).
}

Répartition: Alpes, Mittelgebirge, Angleterre, Laponie suédoise et finnoise (Saether 1968). Il s'agit d'une répartition boréoalpine avec présence relicte dans les Mittelgebirge (Brundin 1956) et le Massif Central français (Verneaux 1968).

La larve, sténotherme d'eau froide, vit dans les sources rhéocrènes (Fittkau 1954).

Sa prédominance dans les 2 stations les plus froides A (la plus proche du névé) et $a 6>$ (source rhéocrène froide) confirme l'écologie de cette espèce.

En a $>$ >ù l'espèce est abondante, la période de vol dure d'août à mi-septembre, ailleurs, les imagos ont surtout été récoltés en octobre. L'Estaragne est la seule station pyrénéenne connue de cette espèce.

63 oิ, 9 ₹.

\section{Paratrichocladius rufiventris (Mg.)}

syn.: Syncricotopus Br. (Hirvenoja 1973).

Répartition : Europe (Lehmann 1971).

Les larves, relativement eurythermes mais rhéophiles-rhéobiontes, vivent de la source au Potamon de la Fulda (Lehmann, loc. cit). Également signalée dans la zone de ressac du lac de Constance (Bodensee) (Reiss 1968). Quelques imagos et nymphes récoltées aux stations $\mathrm{R}, \mathrm{C}$ et a4 fin septembre à mi-uctobre.

6 s, 1 우 $9 \mathrm{~N}$ s, $6 \mathrm{~N}$ ․

\section{Rheocricotopus effusus $\mathrm{K}$.}

Répartition: Europe de l'Ouest, du Centre et de l'Est (Lehmann 1969); Canada (Saether 1973); Jura (Verneaux et Vergon 1976).

La larve rhéobionte et oxybionte vit dans des tubes de vase sur les pierres et les plantes aquatiques de la source à l'Hyporhithron de la Fulda (Lehmann 1971). Dans les Alpes, c'est un élément des sources (Thienemann 1954).

Récoltée de bas en haut du torrent d'Estaragne, $R$. effusus fréquente davantage le petit ruisseau affluent a4 très riche en mousses où sa période de vol va de fin août à fin septembre. Dans les autres stations, les récoltes d'imagos, nettement moins nombreuses, s'échelonnent de mi-juillet à début novembre.

15 ช, 3 \% $13 \mathrm{~N}$ \&, $6 \mathrm{EN}$ \& ; $7 \mathrm{~N}$ \%, $11 \mathrm{EN}$ \%.

\section{Rheocricotopus fuscipes $\mathrm{K}$.}

Répartition: Europe, sauf le Sud (Lehmann 1969); Jura (sub. $R$. dispar G.) (Verneaux et Vergon 1976). 
La larve, eurytherme et eurytope, connue depuis la source jusqu'au Potamon de la Fulda prédomine dans la partie inférieure au niveau du Métarhithron et du Potamon (Lehmann 1971). Neuvième Chironomide par ordre d'importance numérique décroissante du Breitenbach (Ringe 1974).

Récoltée, toujours en petit nombre dans la partie basse de l'Estaragne, jamais en amont de la station $R$.

26 \&, 3 \%; $1 \mathrm{~N} \delta, 4 \mathrm{~N}$ \%.

\section{Metriocnemini}

\section{Chaetocladius laminatus $\mathrm{Br}$.}

Répartition: Alpes françaises, Allemagne, Suède (Lehmann 1971).

Dans la Fulda, depuis la source jusqu'à la zone moyenne à truite (Métarhithron) mais souvent dans les mousses du bord des sources (Lehmann, loc. cit.).

Dans le vallon d'Estaragne, surtout récoltée au niveau de la source rhéocrène $a 6>$ riche en mousses, mais également aux stations $C$ et a4. Imagos le plus souvent capturées en septembre et octobre.

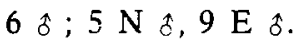

\section{Chaetocladius sp.}

Ce Chaetocladius, caractérisé par l'extrême brachycérie des imagos $\hat{\imath}$, est vraisemblablement nouveau (Reiss, in litt.). Récolté de bas en haut du torrent, il domine à la station C (20\% des récoltes).

Période de vol : août et septembre.

48 o ; $1 \mathrm{~N}$ \&, $2 \mathrm{~N}$ \%.

\section{Corynoneura lobata Edw.}

Répartition et écologie (Schlee 1968): Espèce des eaux courantes froides des cours d'eau des Mittelgebirge centrales et de Forêt Noire. La larve vit le plus souvent dans des sédiments peu profonds. Également connue d'Angleterre. Dans la Fulda, depuis la source jusqu'au Métarhithron (Lehmann 1971).

Déjà signalée dans les Pyrénées, dans le massif de Néouvielle entre $2141 \mathrm{~m}$ et $2215 \mathrm{~m}$ (Laville 1972).

Récoltée de bas en haut du torrent d'Estaragne et de ses affluents, C. lobata colonise aussi bien les stations les plus chaudes (C, a4, a5) que les stations froides ( $\mathrm{a} 6>, \mathrm{A}$ ). Les imagos ont été capturées entre fin juillet et fin octobre, le maximum en août-septembre (en a6 > et a4).

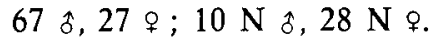

\section{Krenosmittia boreoalpina $\mathrm{G}$.}

Espèce à répartition boréoalpine avec présence relicte dans les 
Mittelgebirge; la larve vit dans les mousses des sources et en faciès hygropétrique de la Laponie aux Alpes (Thienemann 1954) et dans les Carpathes (Albu 1966). Signalée dans le Métarhithron du Rohrwiesenbach en juillet-août, rivière de la Hessen (Ringe 1974) ? Canada (Saether 1969).

D'Est en Ouest de la chaîne pyrénéenne : Méandres d'Estibère $(2050 \mathrm{~m})$ (Laville 1972); deux larves dans le fleuve côtier Tech Pyrénées orientales) (Bertrand $1950 \mathrm{c}$ ); rivière le Lissuraga (Pyrénées atlantiques : Laville, sous presse).

Capturée tout le long du torrent d'Estaragne, $K$. boreoalpina vit surtout au niveau du ruisseau affluent a6 où le maximum des émergences se situe fin juillet à début septembre.

$31 \hat{\circ} ; 12 \mathrm{~N}$ s, $6 \mathrm{~N}$ \% .

\section{Parakiefferiella coronata Edw.}

Répartition: Boréoalpine au sens large (Wülker 1957, Reiss 1968). Cette espèce vit surtout dans la végétation littorale des milieux limniques (Brundin 1949). Elle a été trouvée fréquemment dans la zone littorale des lacs d'altitude pyrénéens (Laville 1972). Elle est rarement signalée en milieu lotique.

Dans l'Estaragne elle fréquente surtout les stations froides (A, a6 $>$, R). 13 imagos ô ont été récoltées au niveau de la station a6 > à la mi-septembre.

15 s., 3 우 $1 \mathrm{~N}$ 。

\section{Paraphaenocladius penerasus Edw.}

Répartition : Europe, Amérique du Nord, Afrique et Australie (Saether 1968). Strenzke (1950) considère les larves du genre Paraphaenocladius comme terrestres. Selon Thienemann (1954) P. impensus est une espèce dont la larve peut vivre dans les mousses des sources et en milieu hygropétrique. Il doit en être de même de $P$. penerasus dont de nombreuses nymphes ont été recueillies à la station a4, la plus riche en mousses. Les autres récoltes proviennent des stations chaudes ( $D$ et a5).

En a4, les imagos ont été récoltées en septembre.

4 o ; $5 \mathrm{~N}$ 3, $9 \mathrm{~N}$ ㅇ.

\section{Chironominae}

\section{Chironomini}

\section{Polypedilum albicorne (Mg.)}

Répartition: Europe du Nord et du Centre (Lehmann 1971); en France: Massif Central (Verneaux 1968), Jura (Verneaux et Vergon 1976). 
Espèce crénophile, sténotherme d'eau froide signalée par Lehmann (loc. cit.) depuis la source jusqu'à l'Epirhithron de la Fulda.

C'est le seul représentant des Chironomini recensé dans le torrent d'Estaragne. Jamais en nombre, il colonise les stations basses du torrent mais aussi le ruisseau affluent froid a $6>$. Déjà signalé dans une source limnocrène à $2100 \mathrm{~m}$ (Laville 1972) et dans la vallée d'Aure à la cressonnière d'Agos (Laville, sous presse).

19 of $2 \mathrm{~N} \delta, 1 \mathrm{~N}$.

\section{Tanytarsini}

\section{Krenopsectra fallax Reiss}

Répartition alpine: Alpes et Pyrénées (Val d'Enfer: Reiss 1969).

Espèce sténotherme d'eau froide vivant dans les mousses des sources et des cours d'eau.

2 \&̊, $2 \mathrm{~N}$ ờ récoltés en août aux stations $\mathrm{B}$ et $\mathrm{R}$.

\section{Lauterbornia coracina $\mathrm{K}$.}

Répartition: Spitzỏerg, Ile aux Ours, Norvège, Suède, Allemagne, Pologne, Suisse (Brundin 1949); Pyrénées (Laville 1966).

Espèce nettement sténotherme froide: vit dans des lacs peu profonds ou des mares de l'Arctique et dans la zone profonde bien oxygénée des lacs à stratification stable d'Europe moyenne (Brundin 1949).

La récolte d'imagos et de nymphes à la station $\mathrm{D}$ (proche du lac d'Orédon), d'imagos seulement aux stations a6 $>$ et a $6<$ (plus éloignées du lac) semble indiquer que la larve peut coloniser des milieux lotiques.

Période de vol de mi-août à octobre.

53 o, 19 ; $; 2 \mathrm{~N}$ ㅇ.

\section{Micropsectra bidentata $G$.}

Répartition: Toute l'Europe (Brundin 1949, Lehmann 1971); en France: Jura (Verneaux et Vergon 1976); lacs du Massif Central (Verneaux 1968); Barèges dans les Hautes-Pyrénées (Bertrand 1950 a et b), dans la cressonnière d'Agos (780 m) et dans les Pyrénées orientales (la Lladure $1480 \mathrm{~m}$ : Laville, sous presse).

Lehmann (loc. cit.) la signale de la source à l'Epirhithron de la Fulda où les larves vivent dans les sédiments d'anses lénitiques.

$M$. bidentata est fréquente de bas en haut du torrent d'Estaragne ( + de $5 \%$ des récoltes). Elle est, avec $D$. hamaticornis et E. tirolensis l'espèce dominante de la station a6 > également bien représentée en $\mathrm{R}$ et a5. On observe un décalage de plus d'un mois entre le début des éclosions imaginales de la station D (mi-juillet) et de la station A 
(début septembre) où les dernières imagos ont été recueillies fin octobre.

201 คิ, 288 ; $60 \mathrm{~N}$ คิ, $13 \mathrm{EN}$ คิ, $80 \mathrm{~N}$ ๆ, $12 \mathrm{EN}$.

\section{2. - ESPËCES SECONDAIRES}

TANyPodinae

Zavrelimyia barbatipes (K.)

Nilotanypus dubius (Mg.)

Pentaneura sp. Katterjokk a6 $<1$ oิ (13.8.71); a5: $1 \mathrm{~N}$ ㅇ $(20$. $8.71)$.

a5: $2 \mathrm{~N}$ के, $2 \mathrm{~N}$ ㅇ (13.8.71); $1 \mathrm{E}$ oै, $1 \mathrm{~N} \hat{\delta}, 2 \mathrm{~N} q(20.8 .71) ; 1 \mathrm{~N}$ ㅇ (27.9.71).

$\mathrm{D}: 1 \mathrm{~N}$ (29.8.72).

\section{ORTHOCLADINAE}

Diamesini

Diamesa incallida (Walk.) $\quad$ R : 1 ô (22.11.76).

Orthocladiini

Prodiamesa ekmani $\mathrm{Br}$.

$\mathrm{D}: 1$ 个.

Prodiamesa olivacea (Mg.)

C: 1 ô (27.9.71).

Brillia longifurca $\mathrm{K}$.

D : 1 के (7.9.71).

Eudactylocladius sp. a6 > : 1 ô (21.7.71).

Eudactylocladius obtexens $\mathrm{Br}$.

C: 2 oे (7.7.71).

Eudactylocladius tuberculatus

C: 1 ô (7.7.71). Edw.

Euorthocladius frigidus (K.) $\quad$ C: $1 \hat{\text { o }}$ (15.9.71); D : 1 ô (29.8.72). a6 $<: 1$ ô (14.8.71).

Orthocladius decoratus $\mathrm{D}: 1$ ô (21.9.71). (Holm.)

Orthocladius excavatus $\mathrm{Br}$. a6 > : 1 of (21.8.71).

Orthocladius rubicundus (Mg.) a5: $1 \mathrm{~N}$ ô (7.9.71).

Microcricotopus bicolor (Zett.) D : 1 ô (7.8.71).

\section{Metriocnemini}

Chaetocladius gracilis $\mathrm{Br}$.

Chaetocladius excerptus (Walk.)

Limnophyes gurgicola Edw. Limnophyes minimus (Mg.)

C: 1 오 (22.10.71).

D : 1 ô (2.10.71).

C : 2 우 (8.8.71); 1 oิ (9.10.71).

$\mathrm{A}: 2$ ô (7.9.71); $\mathrm{B}: 1$ 수 (15.8.71).

D : 1 के (7.9.71); a6 > : 2 के (28.7. 71). a6 $<: 2$ of (7.8.71). 


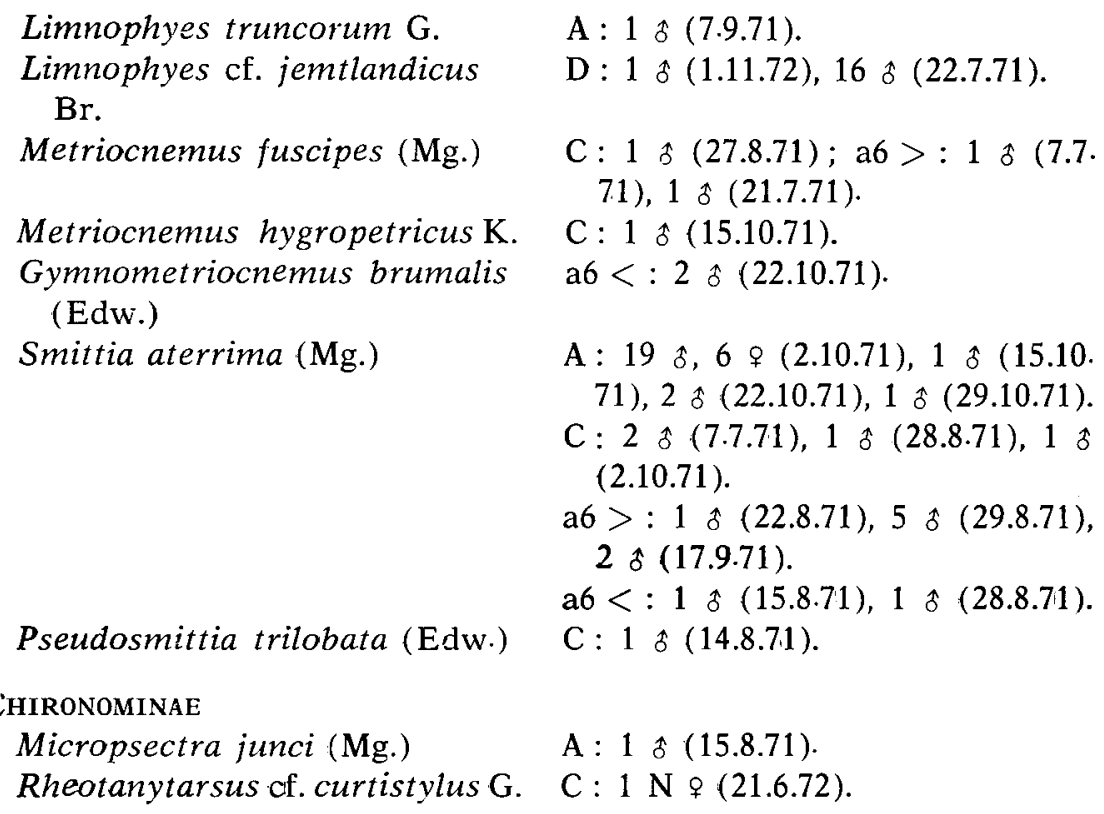

\section{4. - LES BIOCENOSES}

La caractérisation des biocénoses a toujours posé le problème de la représentativité des espèces. Doit-on définir une communauté par la présence constante d'espèces dominantes ou par la présence d'espèces sténotopes? En fait, la simple observation montre qu'une communauté rassemble aussi bien des espèces dominantes constantes que des espèces caractéristiques d'un seul type d'habitat.

En Estaragne, ces biocénoses se répartissent suivant des conditions de milieu essentiellement déterminées par l'altitude, la topographie et le mode d'alimentation des cours d'eau. Au-dessus de $2100 \mathrm{~m}$, le vallon est étroit, encaissé, l'enneigement prolongé (5-7 mois). La proximité du névé d'alimentation impose au torrent des températures toujours froides $\left(<9{ }^{\circ} \mathrm{C}\right)$. L'affluent a4 déneige souvent au début de l'été, mais du fait de sa morphométrie, sa température peut atteindre $15^{\circ} \mathrm{C}$.

Dans la partie basse, le vallon s'ouvre largement à l'Est. L'enneigement est court (3-5 mois). Avec l'éloignement du névé et un meilleur ensoleillement les fluctuations thermiques journalières s'accusent et les maxima atteignent $13{ }^{\circ} \mathrm{C}$ en été au niveau du lac d'Orédon.

L'affluent a5, étroit, bien exposé, est le plus chaud du vallon; par contre, dans l'affluent a6, issu de sources rhéocrènes froides, la température n'excède pas $5^{\circ} \mathrm{C}$ à la station a6 $>$ et $8{ }^{\circ} \mathrm{C}$ en dessous (station $\mathrm{a} 6<$ ). 


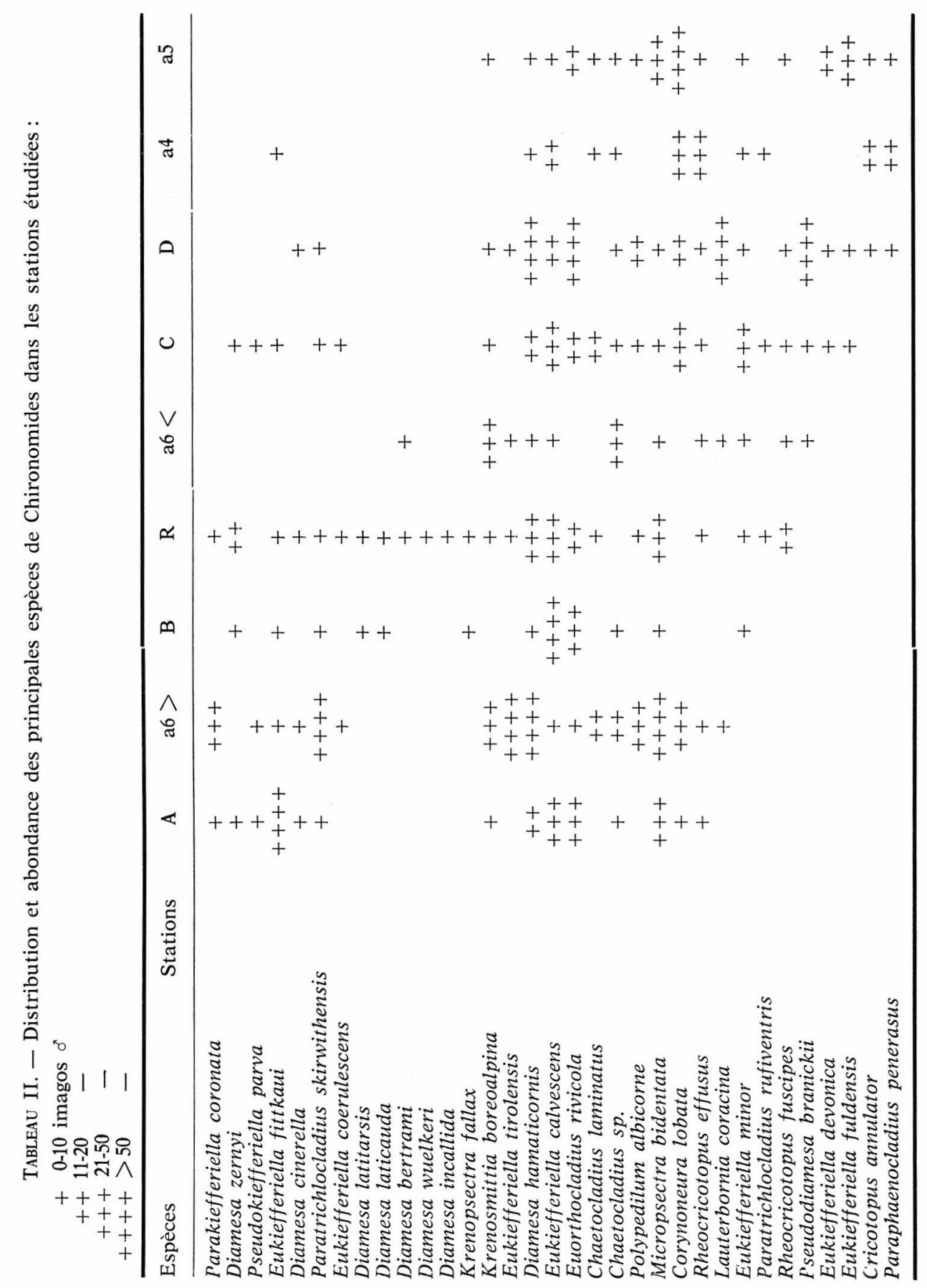




\section{1. - LA PARTIE HAUTE}

Les stations A et B, avec respectivement 14 et 12 espèces, montrent un net appauvrissement de la faune par rapport aux stations inférieures. Elles peuvent être caractérisées par Paratrichocladius skirwithensis, espèce sténotherme fréquente dans cette partie et surtout par Eukiefferiella fittkaui qui prédomine à la station A et peut être considérée comme sténotherme froide.

4 espèces, peu fréquentes, ont surtout été récoltées dans cette zone :

Parakiefferiella coronata (surtout en A)

Diamesa zernyi (A, B, R)

Diamesa cinerella $(\mathrm{A}, \mathrm{R})$

Diamesa laticauda (B, R).

Deux Diamesini des sources froides de haute montagne et des torrents glaciaires ont été capturées à la station $\mathrm{R}$ seulement :

Diamesa wuelkeri

Diamesa bertrami.

Cette station $R$, du fait de son altitude et d'une plus grande variété de biotopes, présente un nombre d'espèces (22) comparable à celui des stations basses.

Diamesa latitarsis, signalée par Serra-Tosio (1973) des sources plus eurythermes et capturée surtout en $\mathbf{B}$, est plus rare en $\mathbf{R}$ et $\mathbf{D}$.

\section{2. - LA PARTIE BASSE}

Les stations basses montrent une plus grande diversité spécifique (21 à 23 espèces) et aussi un plus grand nombre d'espèces " terrestres $»(8-11)$.

Deux espèces peuvent caractériser cette partie :

Eukiefferiella minor (fréquente en $\mathrm{C}$ )

Pseudodiamesa branickii (fréquente en D).

E. minor paraît moins sténotherme que E. fittkaui qui lui succède dans les stations plus froides.

$P$. branickii, signalée pour la première fois dans les Pyrénées, serait une espèce moins montagnarde que Pseudodiamesa nivosa (Pagast 1947) et propre au Crénon (Serra-Tosio 1973). Signalée antérieurement d'une source rhéocrène froide, la tourbière Despax, à $2150 \mathrm{~m}$ (Laville 1966), P. branickii n'a toutefois pas été récoltée en amont de la station C (1920 m).

Quelques espèces, plus rares et davantage eurythermes, n'ont été récoltées que dans la partie basse:

Eukiefferiella devonica

Eukiefferiella fuldensis

Rheocricotopus fuscipes 
Paraphaenocladius penerasus

Cricotopus annulator.

\section{3. - ESPĖCES ABONDANTES DANS L'ENSEMBLE DU TORRENT}

Il existe un groupe d'espèces abondantes qui colonisent l'ensemble du torrent d'Estaragne. Il s'agit à la fois d'espèces eurythermes et eurytopes telles:

Eukiefferiella calvescens

Euorthocladius rivicola

mais aussi, et en plus grand nombre, d'espèces sténothermes froides :

Diamesa hamaticornis

Rheocricotopus effusus

Corynoneura lobata

Chaetocladius $s p$.

Polypedilum albicorne

Micropsectra bidentata.

\section{4. - LA STATION a6>}

La station a6 $>$, affluent du torrent, est une source rhéocrène avec des températures froides comparables à celles des stations hautes; elle tient son originalité d'une abondante couverture de mousses. Cette situation peut expliquer sa plus grande richesse spécifique (18 espèces) et la prédominance d'une espèce caractéristique Eukiefferiella tirolensis dont l'écologie est encore peu connue. Elle vit en grand nombre et presque exclusivement dans cette source froide et peut, ainsi, être considérée comme crénobionte et muscicole.

Le peuplement chironomidien diffère peu, du moins qualitativement, de celui des stations hautes sinon par l'absence de Eukiefferiella fittkaui, Diamesa zernyi, Euorthocladius rivicola et par la présence de Chaetocladius laminatus, espèce muscicole du bord des sources (Lehmann 1971).

La prédominance de Krenosmittia boreoalpina dont l'habitat préférentiel s'avère être les mousses des sources peut servir à caractériser ce biotope.

\section{5. - DISCUSSION}

Cette étude donne une image relativement complète du peuplement Chironomidien d'un torrent froid de haute altitude des Pyrénées. $\mathrm{Si}$ on exclue les espèces terrestres ou hygropétriques (sensu Strenzke 1950), le nombre des Chironomides dont les stades préimaginaux sont aquatiques atteint 33 sur les 58 espèces recensées dans ce travail. 
Parmi celles-ci, la moitié du peuplement est assuré par les Diamesini (10 espèces) et les Eukiefferiella ( 7 espèces) (fig. 2).

Diamesa zemyi
Dicmesa cinerella
Diamesa hamaticomis
Pseudokiefferiella parva
Diamesa laticauda
Diamesa urulkeri
Diamesa incallida
Diamesa latitarsis
Diamesa bertrami
Pseudokiamesa branickii
Eukiefferiella calvescens
Eukiefferiella fittkaui
Eukiefferiella minor
Eukiefferiella coemlescens
Eukiefferiella tirolensis
Eukiefferiella devonica
Eukiefferiella fuldensis

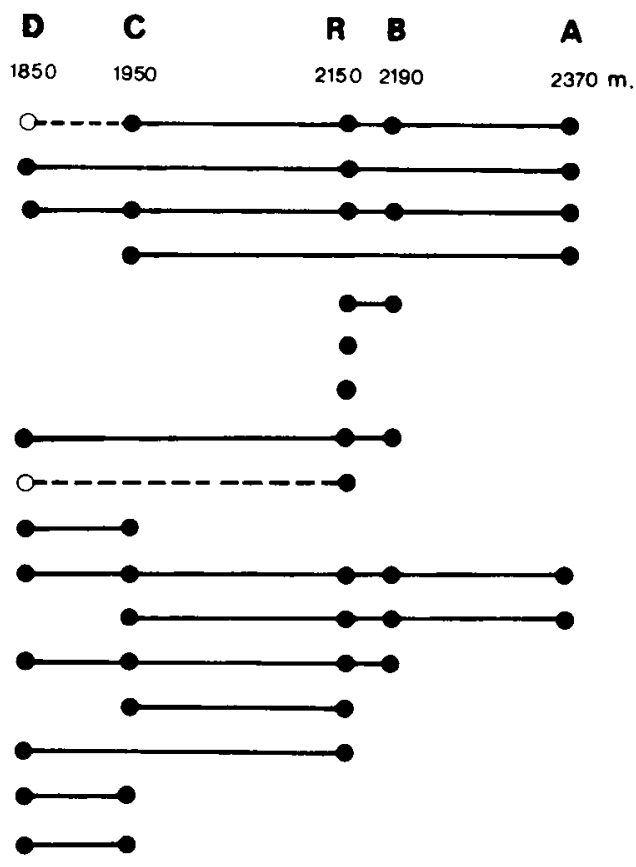

FIG. 2. - Distribution verticale des Diamesini et Eukiefferiella dans Ie torrent d'Estaragne entre $2370 \mathrm{~m}$ et $1850 \mathrm{~m}$. o: récoltes en vol au voisinage de sources tributaires du lac d'Orédon $(1850 \mathrm{~m})$.

Les Diamesini peuplent préférentiellement les eaux courantes froides de 'montagne depuis les sources jusqu'à la zone inférieure à truite : ils colonisent les pierres à couverture biologique plus ou moins dense, certains, notamment Pseudokiefferiella parva et Pseudodiamesa branickii, peuvent également vivre dans les mousses immergées du Crénon (Serra-Tosio 1973). Les espèces du genre Eukiefferiella sont exclusivement rhéophiles, le plus souvent oxybiontes; elles colonisent les mousses aquatiques des eaux à courant rapide bien oxygénées (Lehmann 1972).

Thienemann (1954) a proposé une zonation des cours d'eau de montagne des Alpes basée sur des groupements d'espèces de Chironomides. Il définit successivement le peuplement caractéristique des torrents glaciaires (Gletscherbach), des torrents de haute montagne (Hochgebirgsbach) et des torrents de transition (Übergangsbach).

Le torrent d'Estaragne présente à la fois des caractères physiographiques de torrent glaciaire dans sa partie haute et ceux d'un torrent de haute montagne dans sa partie basse. 
Pour comparer avec les cours d'eau de montagne des Alpes, nous considérons les deux groupes les plus importants du peuplement: Diamesini et Eukiefferiella. Thienemann définit les torrents glaciaires alpins avec 7 Diamesini et 1 Eukiefferiella et les torrents de haute montagne avec 6 Diamesini et 4-5 espèces d'Eukiefferiella.

L'ensemble du torrent d'Estaragne, en fonction de l'importance des espèces récoltées, peut être défini par 6 Diamesini et 5 Eukiefferiella avec prédominance des Diamesini (6) dans la partie froide et inversement des Eukiefferiella (4) dans la partie basse.

Si on compare les listes faunistiques de Thienemann (1954) avec celles de l'Estaragne nous retrouvons 4 des 7 espèces de torrents glaciaires :

- 3 dans la partie haute:

Diamesa cinerella

Diamesa latitarsis

Pseudokiefferiella parva (syn. D. tyrolensis).

- 1 dans la partie basse :

Pseudodiamesa branickii.

L'affinité des deux faunes apparaît mieux si l'on compare les espèces d'Eukiefferiella (tableau III) signalées dans les torrents de haute montagne : 4 espèces de l'Estaragne peuvent être assimilées aux Eukiefferiella des torrents alpins.

Tableau III. - Comparaison des peuplements de système alpin de Thienemann (1954) et du torrent pyrénéen de l'Estaragne.

Torrent de haute montagne (Alpes)

Torrent d'Estaragne (Pyrénées)

(Thienemann :1954)

Eutanytarsus inermipes-group

Micropsectra sp. - - - - - - - - Micropsectra bidentata

Phaenopelma sp.

Diamesa steinböcki

Diamesa zernyi

Diamesa latitarsis _ - $-\ldots+\ldots$ - $-\ldots$ Diamesa latitarsis

Diamesa insignipes Diamesa hamaticornis

Diamesa davisi

Diamesa bertrami

Diamesa $\mathrm{sp.}$

Pseudodiamesa branickii

Diamesa laticauda

Onychodiamesa macronyx

Boreoheptagia sp.

Cardiocladius sp.

Eukiefferiella cf. calvescens-lobulifera - - - Eukiefferiella calvescens

Eukiefferiella sp. cf. minor-montana - - - Eukiefferiella minor, E. fittkaui

Eukiefferiella brevicalar, cf. claripennis

(syn. hospita) et alpestris $-\ldots-\ldots---$ Eukiefferiella fuldensis $(N \neq$ claripennis $)$

Eukiefferiella cf. devonica _ _ _ _ _ _ Eukiefferiella devonica

Parorthocladius nudipennis Eudactylocladius rivicola 
On remarque toutefois l'absence dans l'Estaragne de Diamesa steinböcki plusieurs fois signalée à l'état larvaire par Bertrand $(1950 \mathrm{~b})$. La larve, fréquemment confondue avec celle d'autres Diamesa (Wülker 1959) est, en réalité, proche des larves du groupe cinerella (SerraTosio (1973). Cependant, bon nombre des récoltes de Bertrand proviennent de stations comprises entre $2600 \mathrm{~m}$ et $3000 \mathrm{~m}$, ce qui n'exclue pas complètement la présence de $D$. steinböcki à plus haute altitude dans de véritables sources ou torrents glaciaires. Dans les Alpes, la répartition altitudinale de cette espèce s'étend de $2000 \mathrm{~m}$ à $3000 \mathrm{~m}$ (Serra-Tosio 1973).

Des études récentes de cours d'eau dans des massifs montagneux européens sont surtout consacrées aux Diamesini des Alpes (SerraTosio 1973, Kownacka et Knownacki 1975) et de l'ensemble TatrasCausase-Balkans (Kownacki et Kownacka 1974); des travaux intéressant la faune chironomidienne dans son ensemble sont plus rares et souvent basés sur du matériel larvaire qui, s'il peut apporter une meilleure connaissance de l'écologie des genres ou groupes d'espèces, n'en reste pas moins insuffisant pour une bonne précision systématique. Il est de ce fait difficile de comparer les peuplements spécifiques de torrents d'autres massifs montagneux tels ceux des hauts Tatras, du Caucase et des Balkans étudiés par Kownacki (1971) et Kownacki et Kownacka (1972).

Si l'on se réfère au groupe des Diamesini récemment étudié par ces auteurs (Kownacka et Kownacki 1975) dans un torrent glaciaire des Alpes du Tyrol entre 1400 et $2500 \mathrm{~m}$, nous y trouvons 8 espèces communes sur les 15 répertoriées:

Pseudodiamesa nivosa (source Despax $2150 \mathrm{~m}$ : Laville 1966)

Pseudodiamesa branickii

Diamesa bertrami

Diamesa cinerella

Diamesa incallida

Diamesa latitarsis

Diamesa zernyi

Pseudodiamesa parva.

2 espèces trouvées dans l'Estaragne ne sont pas signalées des Alpes du Tyrol :

Diamesa wuelkeri

Diamesa hamaticornis.

Enfin deux espèces déjà capturées dans le Massif de Néouvielle n'ont pas été retrouvées dans l'Estaragne : Diamesa thomasi Ser.Tos. en mars 1962 à la Neste de Couplan $(1590 \mathrm{~m})$ et Diamesa lavillei Ser.Tos. en mars-avril entre 1100 et $1940 \mathrm{~m}$ ainsi qu'à $2000 \mathrm{~m}$ dans le massif espagnol des Encantats (Serra-Tosio 1973), peut-être en raison de leur précocité. 
La faune du torrent d'Estaragne montre une intrication étroite des formes sténothermes et des formes eurythermes. Les premières (la plupart des Diamesini) peuvent coloniser le torrent jusqu'au lac car le névé d'alimentation et les sources froides affluentes maintiennent de basses températures jusqu'à l'embouchure; les secondes peuvent coloniser l'ensemble du réseau hydrographique en profitant des habitats les plus cléments ou de la saison la plus favorable; elles restent cependant plus abondantes dans la partie basse du torrent (Tableau II).

La faune chironomidienne est diversifiée (58 espèces dénombrées) mais 9 espèces seulement sont numériquement importantes. Cette hétérogénéité du peuplement, caracténistique des milieux extrêmes, semble de règle en Estaragne. Il est toutefois probable que les proportions relatives des espèces dominantes varient d'une année à l'autre, du fait des conditions météorologiques annuelles et du caractère univoltin des cycles biologiques.

\section{TRAVAUX CITÉS}

Albu (P.). 1966. - Chironomide din Carpatii Românesti. ${ }^{\circ}$. Studii Cerc. Biol. Seria Zoologie, 18 (3) : 193-205.

Bertrand (H.). 1950 a. - Chironomides pyrénéens nouveaux pour la faune française. Bull. Soc. ent. Fr., 55 : 11-12.

Bertrand (H.). 1950 b. - Diptères Chironomides pyrénéens. Bull. Soc. ent. Fr., $55: 1531155$.

Bertrand (H.). $1950 \mathrm{c}$. - Récoltes de Diptères Chironomides dans les Pyrénées. Vie et Milieu, 1 (3) : 345-355.

Bertrand (H.). 1952. - Récoltes de Diptères Chironomides dans les Pyrénées. ( $2^{*}$ note.) Vie et Milieu, 3 (3): 314-321.

Bertrand (H.). 1953. - Diptères Chironomides pyrénéens. (2c note.) Bull. Soc. ent. Fr., $58: 76-79$.

Bertrand (H.). 1955 a. - Sur la faune entomologique des Pyrénées. Verh. Internat. Verein. Limnol., 12 : 634-642.

Bertrand (H.). 1955 b. - A propos de tufs à Chironomides. L'Entomologiste, $\mathrm{XI}, 1: 6-7$.

Bertrand (H.). 1956. - Diptères Chironomides pyrénéens et espagnols. Bull. Soc. ent. Fr., 61 : 93-95.

BRUndin (L.). 1949. - Chironomiden und andere Bodentiere der südschwedischen Urgebirgsseen. Ein beitrag zur Kenntnis der bodenfaunistischen Characterzuige schwedischer oligotropher Seen. Rep. Inst. Freshwat. Res. Drottningholm, $30: 1-9.14$.

Brundin (L.). 1956. - Zur Systematik der Orthocladiinae (Dipt. Chironomidae). Rep. Inst. Freshwat. Res. Drottningholm, 37 : 5-185.

DitTMar (H.). 1955. - Ein Sauerlandbach. Untersuchungen an einem WiesenMittelgebirgsbach. Arch Hydrobiol, 50 : 305-552.

FitTKaU (E. J.). 1962. - Die Tanypodinae (Diptera, Chironomidae). Abh. Larvalsyst. Insekten 6: Berlin, 1-453.

Hirvenoja (M.). 1973. - Revision der Gattung Cricotopus van der Wulp und ihrer Verwandten (Diptera, Chironomidae). Ann. Zool. Fennici, 10: 1-363. 
KoWNACKa (M.) et KoWNACKI (A.). 1975. - Glescherbach-Zuckmüken der ötztaler Alpen in Tirol (Diptera : Chironomidae : Diamesinae). Entomologica Germanica, 2 (1) : 35-43.

Kownacki (A.). 1971. - Taxocens of Chironomidae in streams of the Polish High Tatra Mts. Acta Hydrobiol., 13 (4) : 439-464.

KoWNACKA (M.) et KoWNACKI (A.). 1972. - Vertical distribution of zoocenoses in the streams of the Tatra, Caucasus and Balkans Mts. Verh. Int. Verein. Theor. Angew. Limnol., 18: 742-750.

Kownacki (A.) et Kownacka (M.). 1974. - Relation of Chironomidae from Tatra and Caucasus Mts. Ent. Tidskr., 95, suppl. : 129-138.

LaVANDIER (P.). 1974. - Ecologie d'un torrent pyrénéen de haute montagne. I. Caractéristiques physiques. Annls. Limnol., 10 (2) : 173-210.

Lavandier (P.) et MUR (C.). 1974. - Ecologie d'un torrent pyrénéen de haute montagne. II. Caractéristiques chimiques. Annls. Limnol., 10 (3) : 275-309.

Laville (H.). 1966. - Chironomides du Massif de Néouvielle (Pyrénées centrales) (Diptères). Annls. Limnol., 2 (1): 203-216.

Laville (H.). 1972 (1971). - Recherches écologiques sur les Chironomides (Diptera) des lacs du Massif de Néouvielle (Hautes-Pyrénées). Première partie : Systématique, Ecologie, Phénologie. Annls. Limnol., 7 (2): 1173-332.

Laville (H.). Sous presse. - Inventaire des Chironomides connus des Pyrénées en 1976. Universitatis Carolina Biologica, Prague.

LehmanN (J.). 1971. - Die Chironomiden der Fulda (Systematische, ökologische und faunistische Untersuchungen). Arch. Hydrobiol. Suppl. 37 (4): 466-555.

Lehmann (J.). 1969. - Die europäischen Arten der Gattung Rheocricotopus Thien. und Harn. und drei neue Artvertreter dieser Gattung aus der Orientalis (Diptera, Chironomidae). Arch. Hydrobiol., 66 (3) : 348-381.

LehmanN (J.). 1972. - Revision der europäischen Arten (Puppen ồ und Imagines $\left.\sigma^{\circ} \sigma^{\circ}\right)$ der Gattung Eukiefferiella Thienemann. Beit. Ent., Bd $22(7 / 8)$ : 347-405.

Pagast (F.). 1947. - Systematik und Verbreitung der um die Gattung Diamesa gruppierten Chironomides. Arch. Hydrobiol., 41 : 435-596.

REISs (F.). 1968. - Ökologische und systematische Untersuchungen an Chironomiden (Diptera) des Bodensees. Arch. Hydrobiol., 64 (2 et 3): 176-323.

REISs (F.). 1969. - Krenopsectra fallax gen. n. sp. n. (Diptera, Chironomidae) aus den Alpen und Pyrenäen. Ann. Zool. Fennici, $6: 435-442$. Suppl. $45(2 / 3): 212-304$.

RINGE (F.). 1974. - Chironomiden-Emergenz 1970 in Breitenbach und Rohrwiesenbach Schlitzer Produktionsbiologische Studien (10). Arch. Hydrobiol., suppl. $45(2 / 3): 212-304$.

SAether (O.). 1968. - Chironomids of the Finse Area, Norway, with special reference to their distribution in a glacier brook. Arch. Hydrobiol., 64 (4): 426-483.

SAether (O.). 1969. - Some nearctic Podonominae, Diamesinae, and Orthocladiinae (Diptera : Chironomidae). Bull. Fish. Res. Bd Can., 170: 1-154.

SAETHER (O.). 1973. - Four species of Bryophaenocladius Thien., with notes on other Orthocladiinae (Diptera : Chironomidae). Can. Ent., 105: 51-60.

Schrcz (D.). 1968. - Vergleichende Merkmalsanalyse zur Morphologie und Phylogenie der Corynoneura-Gruppe (Diptera, Chironomidae). Stutt. Beitr. Naturkunde, $180: 1-150$.

Serra-Tosio (B.). 1971. - Contribution à l'étude taxonomique, phylogénétique, biogéographique et écologique des Diamesini (Diptera, Chironomidae) d'Europe. Thèse Doctorat d'Etat, 2 vol., 462 p.: $\mathrm{n}^{\circ}$ C.N.R.S. :A.O. 5990.

Serra-Tosio (B.). 1973. - Ecologie et biogéographie des Diamesini d'Europe (Diptera, Chironomidae). Trav. Lab. Hydrobiol. Grenoble, 63 (1971) : 5-175. 
STRENZKe (K.). 1950. - Systematik, Morphologie und ökologie der terrestrischen Chironomiden. Arch. Hydrobiol. Suppl. 18 : 207-414.

ThIENEMANN (A.). 1941. - Lappländische Chironomiden und ihre Wohngewässer. Arch. Hydrobiol. Suppl. 17 : 1-253.

ThienEmann (A.). 1954. - Chironomus. Leben, Verbreitung und wirtschaftliche Bedeutung der Chironomiden. Binnengewässer, $20: 1-834$.

Verneaux (J.). 1968. - Contribution à l'étude des Chironomides (Diptères Nématocères) de la région de Besse-en-Chandesse (Puy-de-Dôme). Annls. Station biol. Besse-en-Chandesse, 3 : 117-146, 3 pl. h.-t.

Verneaux (J.) et Vergon (J.-P.). 1976. - Faune dulçaquicole de Franche-Comté. Sixième partie : Les diptères Chironomides. Ann. Sci. Univ. Besançon (1974), 11 (3) : 179-198.

WÜLKeR (W.). 1957. - Über die Chironomides der Parakiefferiella-Gruppe (Diptera, Tendipedidae, Orthocladiinae). Beitr. Entomol., 7 (3/4): 411-429. 